\title{
Comparação entre alguns processos de oxidação avançados na cinética de degradação do efluente bruto de curtume
}

\section{Comparison among some advanced oxidation processes in the degradation kinetics of the crude tannery effluent}

\author{
Camila Lopes Maler ${ }^{1}$; Keiko Takashima²
}

Resumo

Neste trabalho foi investigada a cinética de degradação do efluente bruto de curtume, coletado no tanque de homogeneização com demanda química de oxigênio equivalente a $3340 \mathrm{mg} \mathrm{L}^{-1}$ e $\mathrm{pH}$ de 8,1 , por processos de oxidação avançados como fotocatálise mediada por semicondutor, fotólise em presença de peróxido de hidrogênio e processo foto-Fenton a $30^{\circ} \mathrm{C}$. Os experimentos foram realizados usando-se o efluente diluído na proporção de 1:50 em água destilada no interior de um reator cilíndrico de parede dupla aberto à atmosfera, variando-se a concentração dos oxidantes. As constantes de velocidade de degradação, $\mathrm{k}_{\mathrm{obs}}$, do efluente, calculadas sob condições de pseudo-primeira ordem, foram maiores para $\mathrm{o}$ processo fotocatalítico em meio de $\mathrm{ZnO}\left(\mathrm{k}_{\mathrm{obs}}=1,23 \times 10^{-1}\right.$ a $\left.2,74 \times 10^{-1} \mathrm{~min}^{-1}\right)$ seguido por $\mathrm{TiO}_{2}\left(0,90 \times 10^{-2}\right.$ $\min ^{-1}$ a $3,35 \times 10^{-2} \mathrm{~min}^{-1}$ ) em pH ca. 7,3 e $30^{\circ} \mathrm{C}$. Tanto a degradação do efluente sob fotólise na presença de $\mathrm{H}_{2} \mathrm{O}_{2}\left(9,36 \times 10^{-3}\right.$ a $\left.7,44 \times 10^{-3} \mathrm{~min}^{-1}\right) \mathrm{em} \mathrm{pH} 6,8$ quanto sob foto-Fenton $\left(7,30 \times 10^{-3}\right.$ a $\left.12,0 \times 10^{-3} \mathrm{~min}^{-1}\right) \mathrm{em}$ $\mathrm{pH} 5,8$ foi significativamente inferior quando comparada ao processo fotocatalítico a $30^{\circ} \mathrm{C}$. A demanda química de oxigênio do efluente foi totalmente removida após $2 \mathrm{~h}$ no processo fotocatalítico a $30^{\circ} \mathrm{C}$. Palavras-chave: Efluente de curtume. Fotocatálise. $\mathrm{TiO}_{2}$. $\mathrm{ZnO}$.

\begin{abstract}
In this work it was investigated the degradation kinetics of the crude tannery wastewater, collected in the homogenized reservoir with a chemical oxygen demand of $3340 \mathrm{mg} \mathrm{L}^{-1}$ at $\mathrm{pH} 8.1$, through the advanced oxidation processes as photocatalysis mediated by semiconductor, photolysis in presence of hydrogen peroxide and photo-Fenton process at $30^{\circ} \mathrm{C}$. The experiments were performed using a 1:50 proportion in distilled water inside the double wall cylindrical reactor opened to the atmospheric air by varying the oxidant concentration. The degradation rate constants, $\mathrm{k}_{\mathrm{obs}}$, determined under pseudofirst order conditions in $\mathrm{pH}$ ca. 7.3 and $30^{\circ} \mathrm{C}$, were higher for the photocatalytic process mediated by $\mathrm{ZnO}\left(\mathrm{k}_{\mathrm{obs}}=1.23 \times 10^{-1}\right.$ to $\left.2.74 \times 10^{-1} \mathrm{~min}^{-1}\right)$ followed by $\mathrm{TiO}_{2}\left(0.90 \times 10^{-2} \mathrm{~min}^{-1}\right.$ to $\left.3.35 \times 10^{-2} \mathrm{~min}^{-1}\right)$. As to for the rate constants under photolysis in presence of $\mathrm{H}_{2} \mathrm{O}_{2}\left(9.36 \times 10^{-3}\right.$ to $\left.7.44 \times 10^{-3} \mathrm{~min}^{-1}\right)$ at $\mathrm{pH}$ ca. 6.8 as well as in photo-Fenton $\left(7.30 \times 10^{-3}\right.$ to $\left.12.0 \times 10^{-3} \mathrm{~min}^{-1}\right)$ at $\mathrm{pH} 5.8$ was significantly lower than photocatalysis at $30^{\circ} \mathrm{C}$. The chemical oxygen demand of the wastewater was completely removed after $2 \mathrm{~h}$ on photocatalytic process at $30^{\circ} \mathrm{C}$.
\end{abstract}

Keywords: Tannery wastewater. Photocatalysis. $\mathrm{TiO}_{2}$. $\mathrm{ZnO}$.

Aluna de graduação em Química, Universidade Estadual de Londrina

2 Professora do Departamento de Química, Universidade Estadual de Londrina; keiko@uel.br 


\section{Introdução}

O curtume é considerado uma das indústrias mais poluentes em termos de volume e complexidade do efluente gerado. A transformação da pele animal em couro exige uma série de tratamentos e uso de grandes quantidades de produtos químicos. É constituída basicamente por três etapas: ribeira, curtimento e acabamento (NAZER; AL-SA'ED; SIEBEL, 2006; COSTA et al., 2008). A ribeira tem por finalidade a limpeza e eliminação de substâncias como pêlos, gordura e sangue que não farão parte do produto manufaturado, bem como preparar a matriz de fibras colagênicas para posterior adição de produtos químicos nas etapas seguintes. $\mathrm{O}$ curtimento é a etapa que envolve a adição de taninos para tornar a pele animal em material estável e imputrescível. O aspecto visual e o aumento do valor comercial são obtidos na etapa de recurtimento através da adição ao couro curtido de corantes, de taninos, sintético e vegetal, de ácidos orgânicos, sais de cromo e de alumínio, etc. Operações como secagem, prensagem e tingimento na etapa de acabamento dão ao couro manufaturado o aspecto definitivo e consumível comercialmente. Todas as etapas produzem efluentes em grande quantidade e dentre estas, a de recurtimento é uma das responsáveis pelo aumento considerável da demanda química de oxigênio do efluente de curtume. (CASSANO et al., 2001).

A geração de grandes quantidades de efluentes contendo resíduos sólidos (fibras, graxa, pêlos, etc.), metais pesados como cromo e sais dissolvidos tem causado problemas e preocupação ambiental quanto ao destino dos mesmos. As técnicas tradicionais de tratamento são eficientes, mas geralmente transferem estes poluentes da fase líquida à sólida, gerando problemas de descarte destes resíduos altamente tóxicos (SCHRANK et al., 2004, 2005).

Os processos de oxidação avançados (POAs) como fotocatálise mediada por semicondutores, fotólise por peróxido de hidrogênio e foto-Fenton são processos físico-químicos baseados na geração de radicais e reações subsequentes, especialmente o radical hidroxila, ${ }^{\circ} \mathrm{OH}$, que destrói não seletivamente as substâncias tóxicas e refratárias por possuir potencial de oxidação relativamente alto de 2,8 V (LEGRINI; OLIVEROS; BRAUN, 1993). Dentre estes, a fotocatálise heterogênea utiliza semicondutores do tipo-n como $\mathrm{TiO}_{2}, \mathrm{ZnO}, \mathrm{Fe}_{2} \mathrm{O}_{3}$, etc. que possuem bandas de valência (bv) completas e bandas de condução (bc) vazias separadas pela energia de band-gap, $\mathrm{E}_{\mathrm{g}}$, de 3,2 eV para $\mathrm{TiO}_{2}$ e de $3,37 \mathrm{eV}$ para $\mathrm{ZnO}$. Ao irradiar o sistema mediado por semicondutor com energia igual ou maior que Eg será gerado o par elétron $\left(\mathrm{e}^{-}\right)$/lacuna $\left(\mathrm{h}^{+}\right)$ (HOFFMAN et al., 1995) como mostrada em (1).

Semicondutor $+\mathrm{h} v \rightarrow \mathrm{e}^{-}+\mathrm{h}^{+}$

A lacuna formada na banda de valência pode reagir tanto com a água (2) quanto com o íon hidroxila (3) adsorvidos na superfície do semicondutor para gerar ${ }^{\bullet} \mathrm{OH}$

$\mathrm{h}^{+}+\mathrm{H}_{2} \mathrm{O}_{(\mathrm{ads})} \rightarrow \cdot \mathrm{OH}+\mathrm{H}$
$\mathrm{h}^{+}+\mathrm{OH}_{(\mathrm{ads})}^{-} \rightarrow \cdot \mathrm{OH}$

O elétron da banda de condução reage com o oxigênio molecular, reduzindo-o ao ânion radical superóxido, $\mathrm{O}_{2}^{\bullet-}$ (4) que, na seqüência, forma o radical hidroperóxido $\mathrm{HO}_{2}^{\bullet-}$ ao ser protonado (5) que, por sua vez, pode formar $\mathrm{H}_{2} \mathrm{O}_{2}$ (6) e sofrer cisão para produzir ${ }^{\bullet} \mathrm{OH}$.

$$
\begin{aligned}
& \mathrm{e}^{-}+\mathrm{O}_{2(\text { ads })} \rightarrow \mathrm{O}_{2}^{\bullet-} \\
& \mathrm{O}_{2}^{\bullet}+\mathrm{H}^{+} \rightarrow \mathrm{HO}_{2}^{\bullet-} \\
& \mathrm{HO}_{2}^{\bullet-}+\mathrm{e}^{-}+\mathrm{H}^{+} \rightarrow \mathrm{H}_{2} \mathrm{O}_{2}
\end{aligned}
$$

$\mathrm{O}$ radical ${ }^{\bullet} \mathrm{OH}$ pode ainda ser produzido a partir da fotólise de peróxido de hidrogênio em 253,7 nm. A absorção de fóton pelo peróxido de hidrogênio promove a cisão homolítica e forma dois radicais hidroxila (7), os quais reagem rapidamente e 
não seletivamente com a maioria dos compostos orgânicos (LEGRINI; OLIVEROS; BRAUN, 1993).

$$
\mathrm{H}_{2} \mathrm{O}_{2}+h v \rightarrow 2{ }^{\circ} \mathrm{OH}
$$

O processo Fenton, outro exemplo de POA, tem sido bastante aplicado na remoção de contaminantes de meios aquáticos, especialmente àqueles contendo compostos recalcitrantes (DANTAS; JOSÉ; MOREIRA, 2003) e tem-se mostrado eficiente no tratamento de efluentes contendo corantes (MERIÇ, KAPTAN; ÖLMEZ, 2004), fármacos (TROVÓ; MELO; NOGUEIRA, 2008) entre outros. Neste método, o radical hidroxila é produzido na solução pela reação entre $\mathrm{Fe}^{2+}$ e $\mathrm{H}_{2} \mathrm{O}_{2}$ de acordo com a equação 8.

$\mathrm{Fe}^{2+}+\mathrm{H}_{2} \mathrm{O}_{2} \rightarrow \mathrm{Fe}^{3+}+\cdot \mathrm{OH}+\mathrm{OH}^{-}$

$\mathrm{O} \mathrm{Fe}^{3+}$ produzido pode reagir com $\mathrm{H}_{2} \mathrm{O}_{2}$ regenerando $\mathrm{Fe}^{2+}$ e dar sequência à reação (9-11) (PÉREZ et al., 2002a; CARNEIRO; NOGUEIRA; ZANONI, 2007).

$$
\begin{aligned}
& \mathrm{Fe}^{3+}+\mathrm{H}_{2} \mathrm{O}_{2} \rightarrow \mathrm{FeOOH}^{2+}+\mathrm{H}^{+} \\
& \mathrm{FeOOH}^{2+} \rightarrow \mathrm{HO}_{2} \cdot+\mathrm{Fe}^{2+} \\
& \mathrm{Fe}_{3+}+\mathrm{HO}_{2} \rightarrow \mathrm{Fe}_{2+}+\mathrm{O}_{2}+\mathrm{H}^{+}
\end{aligned}
$$

Quando o sistema for irradiado com luz UV, a velocidade de degradação de poluentes pode ser aumentada devido ao ataque direto à matéria orgânica por intermediários de ferro de maior valência (PÉREZ et al., 2002b).
Este trabalho tem como objetivo comparar o comportamento cinético de degradação do efluente de curtume, coletado no tanque de homogeneização, por processos fotocatalíticos mediados por $\mathrm{TiO}_{2} \mathrm{e}$ $\mathrm{ZnO}$; pelo sistema $\mathrm{H}_{2} \mathrm{O}_{2} / \mathrm{UV}$ e pelo processo fotoFenton e avaliar a eficiência do processo através da medida da demanda química de oxigênio.

\section{Materiais e métodos}

O efluente bruto de curtume foi coletado do tanque de homogeneização, formado pela mistura dos efluentes produzidos em todas as etapas de produção do couro, de um curtume localizado na região Norte do Estado do Paraná. O efluente foi filtrado e diluído em água destilada na proporção de 1:50, cujo espectro é ilustrado na Figura 1.

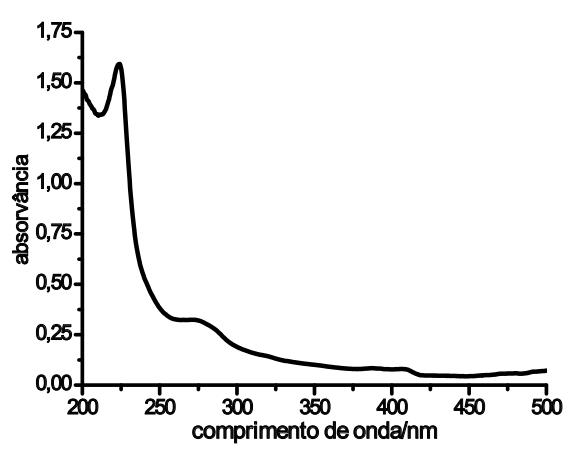

Figura 1. Espectro UV-Vis do efluente diluído em água $(1: 50)$.

O efluente diluído foi irradiado em reator de vidro borossilicato de parede dupla para permitir circulação de água a $(30,0 \pm 0,1){ }^{\circ} \mathrm{C}$ (Microquímica MQ8TC 99-20), usando a lâmpada de vapor de $\mathrm{Hg}$ (125 W) sem bulbo com irradiância de $(1050 \pm 20)$ $\mu \mathrm{W} \mathrm{cm} \mathrm{cm}^{-2}$ (Topcon UVR-2), sob agitação magnética, a $600 \mathrm{rpm}$ (Fisatom 752). 
Os semicondutores $\mathrm{TiO}_{2}$ (Degussa-P25 principalmente anatase, $50 \mathrm{~m}^{2} \mathrm{~g}^{-1}$ não poroso) e $\mathrm{ZnO}$ (Colleman, P.A.) foram utilizados para fotocatálise heterogênea; $\mathrm{H}_{2} \mathrm{O}_{2}(30 \%$, P.A. Merck) previamente padronizado para fotólise e para foto-Fenton, adicionou-se, além de $\mathrm{H}_{2} \mathrm{O}_{2}$, a solução de $\mathrm{Fe}_{2} \mathrm{SO}_{4} \cdot 7 \mathrm{H}_{2} \mathrm{O}$ (Vetec, P.A.). A suspensão formada pelo efluente e semicondutor $\left(\mathrm{TiO}_{2}\right.$ ou $\left.\mathrm{ZnO}\right)$ foi previamente agitada no escuro antes da irradiação. Alíquotas de 1,0 mL foram removidas em tempos pré-determinados e armazenadas em tubos reacionais. $\mathrm{O}$ espectro UV-Vis foi monitorado de 200 a $450 \mathrm{~nm}$ (Ocean Optics CHEM 2000-UV-VIS). A demanda química de oxigênio (DQO) foi determinada pelo método colorimétrico (APHA, 2005) e o carbono orgânico total (COT) no analisador de carbono orgânico total (Shimadzu TOC 5000A). Antes do tratamento, o efluente apresentou DQO de $3340 \mathrm{mg} \mathrm{L}^{-1}$ e COT de 736,7 $\mathrm{mg} \mathrm{L}^{-1}$ e pH 8,1. A constante de velocidade de degradação, $\mathrm{k}_{\mathrm{obs}}$, dos processos de oxidação avançados investigados neste trabalho, foi determinada sob condições de pseudo- $1^{\mathrm{a}}$ ordem, ou seja, com grande excesso de oxidante sobre efluente e calculada graficamente a partir do ln da absorvância em função do tempo de irradiação.

\section{Resultados e Discussão}

A suspensão formada pelo efluente diluído (1:50) e $\mathrm{TiO}_{2}$ ou $\mathrm{ZnO}$ foi agitada no escuro a 30 oC até a absorvância com máximo em $226 \mathrm{~nm}$ se tornar constante em função do tempo. Esta etapa é essencial à fotocatálise, desde que a geração do radical hidroxila ocorre a partir das espécies adsorvidas na superfície do semicondutor. O equilíbrio de adsorção-dessorção atingiu em 45 min tanto na presença de $\mathrm{TiO}_{2}$ quanto de $\mathrm{ZnO}$ (Figura 2) usando-se 1,0 $\mathrm{g} \mathrm{L}^{-1}$ para ambos os semicondutores.

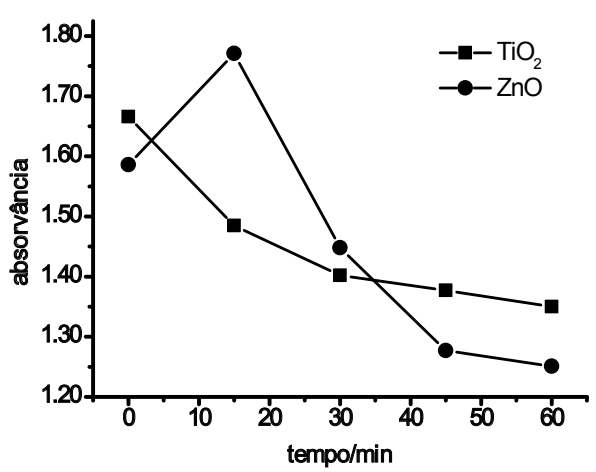

Figura 2. Absorvância máxima $(226 \mathrm{~nm})$ do efluente diluído (1:50) no escuro da suspensão formada por $\mathrm{TiO}_{2}$ $\left(1,0 \mathrm{~g} \mathrm{~L}^{-1}\right)$ ou $\mathrm{ZnO}\left(1,0 \mathrm{~g} \mathrm{~L}^{-1}\right)$ em função do tempo a $30^{\circ} \mathrm{C}$.

Uma vez atingido esse equilíbrio, irradiou-se por $2 \mathrm{~h}$ a suspensão formada pelo semicondutor e efluente por $30^{\circ} \mathrm{C}$ variando-se respectivamente o $\mathrm{TiO}_{2}$ de 0,50 a $3,00 \mathrm{~g} \mathrm{~L}^{-1}$ e o $\mathrm{ZnO}$ de 1,00 a $2,00 \mathrm{~g} \mathrm{~L}^{-1}$ para estudar o efeito da variação de concentração na constante de velocidade de degradação, do efluente como apresentado na Tabela 1. 
Tabela 1. Constante de velocidade de degradação, $\mathrm{k}_{\mathrm{obs}}$, do efluente de curtume (1:50) fotocatalisado por $\mathrm{TiO}_{2}$ e $\mathrm{ZnO}$ em $\mathrm{pH}$ ca. 7,3 e $30^{\circ} \mathrm{C}$.

\begin{tabular}{lcc}
\hline Semicondutor & Concentração/g $\mathbf{L}^{-\mathbf{1}}$ & $\mathbf{k}_{\text {obs }} / \mathbf{1 0}^{-\mathbf{2}} \mathbf{~ m i n}^{\mathbf{- 1}}$ \\
\hline \multirow{2}{*}{$\mathrm{TiO}_{2}$} & 0,50 & 0,90 \\
& 0,75 & 1,64 \\
& 1,00 & 1,61 \\
& 1,25 & 2,01 \\
& 1,50 & 3,35 \\
& 2,75 & 3,15 \\
$\mathrm{ZnO}$ & 3,00 & 2,19 \\
\hline & 1,00 & 23,0 \\
& 1,25 & 24,7 \\
& 1,50 & 27,4 \\
& 1,75 & 13,6 \\
& 2,00 & 12,3
\end{tabular}

Conforme esta tabela, os valores relativamente baixos das constantes de velocidade de 0,50 a 1,25 $\mathrm{g} \mathrm{L}^{-1}$ em suspensão de $\mathrm{TiO}_{2}$ foram atribuídos à pequena disponibilidade de sítios ativos para reagir com os contaminantes. Por outro lado, quando foram variadas de 1,50 a $2,75 \mathrm{~g} \mathrm{~L}^{-1}$ a constante de velocidade atingiu o valor máximo de 3,35×10$2 \mathrm{~min}^{-1}$ em $\mathrm{pH}$ ao redor de 7,3 a $30^{\circ} \mathrm{C}$. Estes resultados mostraram que a maior disponibilidade de sítios ativos, ou seja, de lacunas na banda de valência promoveu a oxidação das moléculas de contaminante, aumentando a eficiência de degradação. A elevação da concentração para 3,0 g $\mathrm{L}^{-1}$ de $\mathrm{TiO}_{2}$ diminuiu o valor de $\mathrm{k}_{\text {obs }}$ para $2,19 \times 10^{-}$ ${ }^{2} \mathrm{~min}^{-1}$. Isto mostra que, o aumento do número de partículas em suspensão pode obstruir a passagem de radiação UV, impedindo e dificultando a formação do par elétron-lacuna para promover a oxidação dos contaminantes.

É sabido que o óxido de zinco apresenta o mecanismo de formação de espécies reativas de forma similar ao $\mathrm{TiO}_{2}$ (CHAKRABARTI; DUTTA, 2004; BEHNAJADY; MODIRSHAHLA; HAMZAVI, 2006; HASNAT et al., 2007). Assim, a concentração de $\mathrm{ZnO}$ foi variada para determinar a rapidez com que ocorre a degradação do efluente, variando-se de 1,00 a 2,00 $\mathrm{g} \mathrm{L}^{-1}$ como mostrado na Tabela 1. Observam-se nesta tabela que as constantes de velocidade são sistematicamente maiores quando comparadas aos valores em meio de $\mathrm{TiO}_{2}$. Segundo Sakthivel et al. (2003) o $\mathrm{ZnO}$ absorve de aproximadamente 470 a $350 \mathrm{~nm}$, atribuída ao processo de transferência de carga da banda de valência à banda de condução, enquanto o $\mathrm{TiO}_{2}$ de 420 a 400 nm, sugerindo que a excitação do elétron ocorra nessa região tanto para $\mathrm{ZnO}$ quanto para $\mathrm{TiO}_{2}$.

Deve-se salientar ainda que o $\mathrm{pH}$ na suspensão tanto em $\mathrm{TiO}_{2}$ quanto em $\mathrm{ZnO}$ diminuiu de 8,1 para cerca de 7,3 e manteve-se praticamente constante 
durante a irradiação. Deste modo, o $\mathrm{TiO}_{2}$ ocorre na forma de $\mathrm{TiO}^{-}$, porque este $\mathrm{pH}$ é superior ao de carga zero $(6,5)$ (HASNAT et al., 2007) e em meio de $\mathrm{ZnO}$ como $\mathrm{ZnOH}^{+}$, pois a carga zero de ZnO ocorre em 11,11 (CHERGINETS; REBROVA, 2006). Isto significa que tanto a reatividade entre $\mathrm{ZnO}$ e o efluente quanto a constante de velocidade são aumentadas em função da maior interação eletrostática promovida pelos eletrólitos presentes. Por outro lado, a variação de $\mathrm{k}_{\mathrm{obs}}$ com aumento da concentração de $\mathrm{ZnO}$ mostrou comportamento similar ao $\mathrm{TiO}_{2}$, ou seja, atingindo valor máximo e posteriormente, diminuindo. Neste caso a constante de velocidade máxima de $27,4 \times 10^{-1} \mathrm{~min}^{-1}$ ocorreu para $1,50 \mathrm{~g} \mathrm{~L}^{-1}$ de $\mathrm{ZnO}$ como mostrado na Tabela 1. $\mathrm{O}$ aumento para $1,75 \mathrm{~g} \mathrm{~L}^{-1}$ diminuiu a constante de velocidade por, pelo menos, $50 \%\left(1,23 \times 10^{-1} \mathrm{~min}^{-1}\right)$, atribuído à saturação dos sítios ativos de modo mais rápido que em $\mathrm{TiO}_{2}$.

A demanda química de oxigênio (DQO) do efluente foi determinada para concentrações de fotocatalisadores que apresentaram os maiores valores de constante de velocidade (Tabela 1), ou seja, 1,50 $\mathrm{g} \mathrm{L}^{-1}$ de $\mathrm{TiO}_{2}$ e 1,75 $\mathrm{g} \mathrm{L}^{-1}$ de $\mathrm{ZnO}$ respectivamente em $\mathrm{pH}$ ao redor de 7,3 a $30^{\circ} \mathrm{C}$. A DQO inicial de $3340 \mathrm{mg} \mathrm{L}^{-1}$ foi completamente removida após 120 min de irradiação em ambos os casos.

Adegradação do efluente foi também investigada por fotólise em presença de peróxido de hidrogênio, variando-se a concentração de 10 a $30 \mathrm{mg} \mathrm{L}^{-1} \mathrm{em} \mathrm{pH}$ ao redor de 6,8 a $30^{\circ} \mathrm{C}$ como apresentada na Figura 3. As constantes de velocidade, calculadas após 120 min de irradiação, foram cerca de 5 a 20 vezes menores em relação ao processo fotocatalítico.

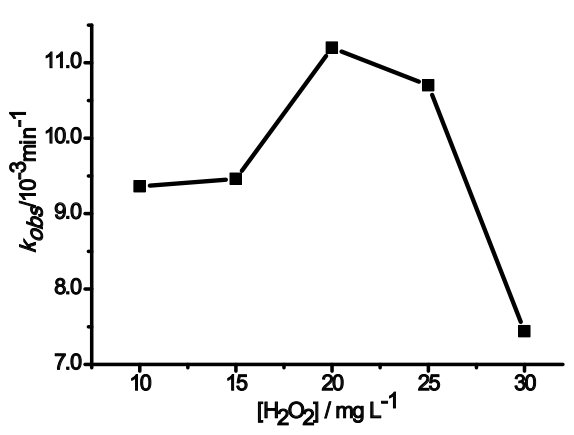

Figura 3: Constante de velocidade de degradação (kobs) do efluente bruto de curtume diluído (1:50) em função da concentração de $\mathrm{H}_{2} \mathrm{O}_{2}$ sob radiação UV em pH 6,8 a $30^{\circ} \mathrm{C}$.

A partir desta figura, observou-se que o efeito da concentração de peróxido de hidrogênio na constante de velocidade seguiu a mesma tendência dos semicondutores. Assim, os maiores valores de kobs ocorreram para 20,0 e $25,0 \mathrm{mg} \mathrm{L}^{-1}$ de $\mathrm{H}_{2} \mathrm{O}_{2}$ que resultaram respectivamente em $11,2 \times 10^{-3}$ e $10,7 \times 10^{-}$ ${ }^{3} \mathrm{~min}^{-1}$. Por outro lado, o aumento para $30,0 \mathrm{mg}$ $\mathrm{L}^{-1}$ diminuiu a constante de velocidade para $7,4 \times 10^{-}$ ${ }^{3} \mathrm{~min}^{-1}$. Atribuiu-se que, em concentrações mais elevadas, $\mathrm{o}_{2} \mathrm{O}_{2}$ tenha atuado como sequestrador de ${ }^{\bullet} \mathrm{OH}$ para produzir o radical hidroperoxila, $\mathrm{HO}_{2}{ }^{\bullet}$, conforme (12)

$$
\mathrm{HO} \bullet+\mathrm{H}_{2} \mathrm{O}_{2} \rightarrow \mathrm{HO}_{2}^{\bullet}+\mathrm{H}_{2} \mathrm{O}
$$

Este radical pode ter provocado a diminuição da eficiência de degradação por ser pouco reativo. Além disso, as constantes de velocidade mais baixas podem ser atribuídas ao fato de neste processo o radical $\bullet^{\bullet} \mathrm{OH}$ ser gerado pela cisão homolítica de $\mathrm{H}_{2} \mathrm{O}_{2}$ (7), com dependência do $\mathrm{pH}$ e ser mais favorável 
quanto mais alcalino seja o meio. Considerando-se que o rendimento quântico em 253,7 nm seja 0,98 (LEGRINI; OLIVEROS; BRAUN, 1993), a baixa eficiência deste processo é decorrente do fato de a lâmpada de mercúrio emitir radiação em torno de $365 \mathrm{~nm}$. Assim, a absorvância inicial do efluente de 1,593 diminuiu 30\% em 120 min ao redor de $\mathrm{pH}$ 6,8 a $30^{\circ} \mathrm{C}$ na degradação mais rápida, ou seja, quando se usou $20 \mathrm{mg} \mathrm{L}^{-1}$ de $\mathrm{H}_{2} \mathrm{O}_{2}$ sob irradiação UV.

Há poucos trabalhos na literatura sobre o tratamento de efluentes de curtume que utilizam o sistema $\mathrm{H}_{2} \mathrm{O}_{2} / \mathrm{UV}$. Por exemplo, Schrank et al. (2004) removeram cerca de $60 \%$ de COT do valor inicial de $45 \mathrm{mg} \mathrm{L}^{-1}$ utilizando-se $400 \mathrm{mg} \mathrm{L}^{-1}$ de $\mathrm{H}_{2} \mathrm{O}_{2}$ em $2 \mathrm{~h}$ de reação. Em concentrações superiores de $\mathrm{H}_{2} \mathrm{O}_{2}$, a degradação foi menos efetiva ( $\left.20-50 \%\right)$. Sauer et al. (2005) determinaram a taxa de remoção máxima equivalente a $0,95 \mathrm{mg} \mathrm{DQO} / \mathrm{L} \min$ da demanda química de oxigênio inicial de $801 \mathrm{mg} \mathrm{O}_{2}$ $\mathrm{L}^{-1}$ usando $500 \mathrm{mg} \mathrm{L}^{-1}$ de $\mathrm{H}_{2} \mathrm{O}_{2}$ em $\mathrm{pH} 2,5$.

Por último, o efeito das concentrações de peróxido de hidrogênio e de sulfato de ferro (II) no processo foto-Fenton foi investigado a $30{ }^{\circ} \mathrm{C}$ e apresentado na Tabela 2, cujas constantes de velocidade foram similares à fotólise na presença de peróxido de hidrogênio.

Tabela 2. Constante de velocidade de degradação, $\mathrm{k}_{\mathrm{obs}}$, do efluente diluído (1:50) obtido por processo foto-Fenton em $\mathrm{pH} 5,8$ a $30^{\circ} \mathrm{C}$.

\begin{tabular}{cccc}
\hline$\left[\mathbf{H}_{2} \mathbf{O}_{2}\right] / \mathbf{m g ~ L}^{-1}$ & {$\left[\mathbf{F e S O}_{\mathbf{4}}\right] / \mathbf{m g ~ L}^{-1}$} & $\mathbf{k}_{\text {obs }^{\prime}} / \mathbf{1 0}^{-3} \mathbf{~ m i n}^{-1}$ & $\mathbf{r}$ \\
\hline 7,0 & 5,0 & 8,10 & 0,988 \\
8,0 & 5,0 & 7,30 & 0,992 \\
9,0 & 4,0 & 7,29 & 0,981 \\
12,0 & 5,0 & 9,51 & 0,996 \\
15,0 & 5,0 & 12,16 & 0,995 \\
\hline
\end{tabular}

As concentrações de peróxido de hidrogênio e de sulfato de ferro (II) usadas neste trabalho foram baseadas em artigos com tratamentos similares com faixa de $\mathrm{pH}$ de 2,5 a 3,5 (DANTAS; JOSÉ; MOREIRA, 2003; MERIÇ; KAPTAN; ÖLMEZ, 2004; SCHRANK et al., 2005). Ao serem testados, observou-se o aumento inesperado da absorvância em toda região espectral UV-Vis. Isto foi atribuído à presença de grande quantidade de substâncias orgânicas em $\mathrm{pH}$ inicial de 8,1. Desta forma, as condições experimentais foram alteradas como apresentado na Tabela 3 . As constantes de velocidade mantiveram-se na mesma ordem de grandeza do processo $\mathrm{H}_{2} \mathrm{O}_{2} / \mathrm{UV}$ e foi mais rápida quando se usou
$15,0 \mathrm{mg} \mathrm{L}-1$ de $\mathrm{H}_{2} \mathrm{O}_{2}$ e $5,0 \mathrm{mg} \mathrm{L}{ }^{-1}$ de $\mathrm{FeSO}_{4} \cdot 7 \mathrm{H}_{2} \mathrm{O}$ em pH 5,8.

De modo geral, a degradação de uma amostra sintética tem mostrado eficiência quando realizado entre $\mathrm{pH}$ 3,0 e 4,0, porque o sistema redox formado pelos íons ferro e peróxido de hidrogênio é mais estável nesta faixa (MERIÇ; KAPTAN; ÖLMEZ, 2004). No entanto, neste intervalo de $\mathrm{pH}$ constatouse o desaparecimento da banda de absorção com máximo em $226 \mathrm{~nm}$ e dando lugar à absorção contínua e superior a 1,8 de 200 a $350 \mathrm{~nm}$. Este comportamento persistiu durante a irradiação da amostra, indicando a formação de compostos recalcitrantes. Desta forma, não foi determinada 
a remoção de DQO em nenhuma das condições investigadas.

O processo foto-Fenton foi empregado por Schrank et al. (2005) que removeram cerca de $60 \%$ da DQO inicial de $130 \mathrm{mg} \mathrm{O}_{2} \mathrm{~L}^{-1}$ em $120 \mathrm{~min}$, quando se utilizou $100 \mathrm{mg} \mathrm{L}^{-1}$ de $\mathrm{H}_{2} \mathrm{O}_{2}$ e $150 \mathrm{mg}$ $\mathrm{L}^{-1}$ de $\mathrm{Fe}^{2+}$ em pH 3,5. A utilização de $\left[\mathrm{Fe}^{2+}\right]=$ $1,0 \mathrm{~g} \mathrm{~L}^{-1}$ e $\left[\mathrm{H}_{2} \mathrm{O}_{2}\right]=15 \mathrm{~g} \mathrm{~L}-1 \mathrm{em} \mathrm{pH}=2,5$ reduziu aproximadamente $90 \%$ da DQO inicial de $1803 \mathrm{mg}$ $\mathrm{O}_{2} \mathrm{~L}^{-1}$ em 240 min de irradiação por Dantas, José e Moreira (2003). Da mesma forma, Lofrano et al. (2007) reduziram 90\% da DQO inicial de $300 \mathrm{mg}$ $\mathrm{L}^{-1}$ em 30 min no tratamento de taninos sintéticos e óleos utilizados na etapa de recurtimento do couro utilizando a razão $\mathrm{H}_{2} \mathrm{O}_{2} / \mathrm{FeSO}_{4}$ igual a 600/500 (mg/ $\mathrm{mg}$ ) em pH 3,5.

\section{Conclusão}

Neste trabalho avaliou-se a fotodegradação do efluente bruto de curtume por alguns processos de oxidação avançados. O efluente foi mais rapidamente degradado por óxido de zinco, seguido por dióxido de titânio em $\mathrm{pH} 7,3$ e $30^{\circ} \mathrm{C}$, usando-se $1,5 \mathrm{~g} \mathrm{~L}^{-1}$ para ambos os semicondutores e cujas DQOs totalmente removidas após 120 min de irradiação. As constantes de velocidade de degradação do efluente obtidas a partir da fotólise em presença de peróxido de hidrogênio e foto-Fenton apresentaram valores inferiores de, pelo menos, 30 vezes em relação ao processo fotocatalítico.

\section{Agradecimentos}

As autoras agradecem ao $\mathrm{CNPq}$ pelo auxílio financeiro e C.L.M. agradece a bolsa de Iniciação Científica PIBIC/CNPq.

\section{Referências}

APHA; AWWA; WEF. Standard methods for the examination of water and wastewater. 21st Edition, Washington: American Public Health Association, 1082 p., 2005.

BEHNAJADY, M. A.; MODIRSHAHLA, N.; HAMZAVI, R. Kinetic study on photocatalytic degradation of C.I. Acid Yellow 23 by $\mathrm{ZnO}$ photocatalyst. Journal of Hazardous Materials, Amsterdam, v. 133, n. 1-3, p. 226-232, 2006.

CARNEIRO, P. A.; NOGUEIRA, R. F. P.; ZANONI, M. V. B. Homogeneous photodegradation of C.I. Reactive Blue 4 using a photo-Fenton process under artificial and solar irradiation. Dyes and Pigments, London, v. 74, n. 1, p. 127-132, 2007.

CASSANO, A.; MOLINARI, R.; ROMANO, M.; DRIOLI, E. Treatment of aqueous effluents of the leather industry by membrane processes: a review. Journal of Membrane Science, Amsterdam, v. 181, n. 1, p. 111-126, 2001.

CHAKRABARTI, S.; DUTTA, B. K. Photocatalytic degradation of model textile dyes in wastewater using $\mathrm{ZnO}$ as semiconductor catalyst. Journal of Hazardous Materials, Amsterdam, v.112, n. 3, p. 269-278, 2004.

CHERGINETS, V. L.; REBROVA, T. P. Solubilities of zinc oxide and cadmium oxide in $\mathrm{KCl}-\mathrm{LiCl}$ eutectic melts. Russian Journal of Inorganic Chemistry, London, v. 51, n. 3, p. 448-487, 2006.

COSTA, C. R.; BOTTA, C. M. R.; ESPINDOLA, E. L. G.; OLIVI, P. Electrochemical treatment of tannery wastewater using DSA ${ }^{\circledR}$ electrodes. Journal of Hazardous Materials, Amsterdam, v. 153, n. 1-2, p. 616-627, 2008.

DANTAS, T. L. P.; JOSÉ, H.; MOREIRA, R. F. P. M. Fenton and photo-Fenton oxidation of tannery wastewater. Acta Scientiarum: Technology, Maringá, v. 25, n. 1, p. 91-95, 2003.

HASNAT, M. A.; UDDIN, M. M.; SAMEDA, A. J. F.; ALAMA, S. S.; HOSSAIN, S. Adsorption and photocatalytic decolorization of a synthetic dye erythrosine on anatase $\mathrm{TiO}_{2}$ and $\mathrm{ZnO}$ surfaces. Journal of Hazardous Materials, Amsterdam, v. 147, n. 1-2, p. 471-477, 2007.

HOFFMANN, M. R.; MARTIN, S. T.; CHOI, W.; BAHNEMANN, D. W. Environmental applications of semiconductor photocatalysis. Chemical Reviews, Washington, v. 95, n. 1, p. 69-96, 1995.

LEGRINI, O.; OLIVEROS, E.; BRAUN, A.M. Photochemical processes for water treatment. Chemical 
Reviews, Washington, v. 93, n.3, p. 671-698, 1993.

LOFRANO, G.; MERIÇ, S.; BELGIORNO, V.; NAPOLI, R. M. A. Fenton's oxidation of various-based tanning materials. Desalination, Amsterdam, v. 211, n. 1-3, p. 10-21, 2007.

MERIÇ, S.; KAPTAN, D.; ÖLMEZ, T. Color and COD removal from wastewater containing Reactive Black 5 using Fenton's oxidation process. Chemosphere, Oxford, v. 54, n. 3, p. 435-441, 2004.

NAZER, D. W.; AL-SA'ED, R. M.; SIEBEL, M. A. Reducing the environmental impact of the unhairingliming process in the leather tanning industry. Journal of Cleaner Production, Amsterdam, v. 14, n.1, p. 65-74, 2006.

PÉREZ, M.; TORRADES, F.; DOMÈNECH, X.; PERAL, J. Fenton and photo-Fenton oxidation of textile effluents. Water Research, New York, v. 36, n. 11, p.2703-2710, 2002a.

. Removal of organic contaminants in paper pulp treatment effluents under Fenton and photoFenton conditions. Applied Catalysis B: Environmental, Amsterdam, v. 36, n. 1, p. 63-74, 2002 b.

SAUER, T.; CASARIL, L.; OBERZINER, A. L. B.; JOSE, H. J.; MOREIRA, R. F. P. M. Advanced oxidation process applied to tannery wastewater containing direct black 38: elimination and degradation kinetics. Journal of Hazardous Materials, Amsterdam, v. 135, n.1-3, p. 274-279, 2005.

SCHRANK, S. G.; JOSE, H. J.; MOREIRA, R. F. P. M.; SCHRODER, H. F. Elucidation of the behavior of tannery wastewater under advanced oxidation conditions. Chemosphere, Oxford, v. 56, n. 5, p. 411-423, 2004.

. Applicability of Fenton and $\mathrm{H}_{2} \mathrm{O}_{2} / \mathrm{UV}$ reactions in the treatment of tannery. Chemosphere, Oxford, v. 60, n. 5, p. 644-655, 2005.

TROVÓ, A. G.; MELO, S. A. S.; NOGUEIRA R. F. P. Photodegradation of the pharmaceuticals amoxicillin, bezafibrate and paracetamol by the photo-Fenton process-Application to sewage treatment plant effluent. Journal of Photochemistry and Photobiology A: Chemistry, Lausanne, v. 198, n. 2-3, p. 215-220, 2008.

Recebido em 25 Fevereiro 2011- Received on February 25, 2011.

Aceito em 13 Maio, 2011 - Accepted on May 13, 2011. 
